\title{
THE FICTION OF THE "GERSTMANN SYNDROME"
}

\author{
BY
}

\section{ARTHUR L. BENTON}

From the Departments of Neurology and Psychology, University of Iowa, Iowa City, Iowa, U.S.A.

The problem with which this study is concerned can be stated as follows. A patient with parietooccipital disease may show one or more of a relatively large number of diverse behavioural deficits. When he presents with two, three, or more of these symptoms, the latter may be viewed by the clinical observer as forming a naturally occurring combination of deficits and given the status of a syndrome, this status implying that the concurrence of deficits is not a chance one, that there is an underlying factor responsible for it, and that it possesses a distinctive neuropathological significance. Once such a special combination or syndrome is established, not only is it used in the observation and description of subsequent cases but it may also determine which aspects of a patient's behaviour are selected for study and which are not. The "Gerstmann syndrome" represents such a combination of behavioural deficits. The study to be reported examines the question of whether, as is usually assumed, this "assembly of unlikely and unexpected symptoms" (Critchley, 1953) is a naturally occurring combination or whether it is only one of a very large number of more or less fortuitous combinations of behavioural deficits which may be encountered in patients with cerebral disease.

The history of the Gerstmann syndrome has been recounted in detail elsewhere (cf. Critchley, 1953; Benton, 1959); only a few major points need be mentioned to introduce the present investigation. Before the end of the nineteenth century, the four behavioural deficits comprising the syndromeright-left disorientation, acalculia, agraphia, and finger agnosia-had been described as occurring in patients with cerebral disease. The first three were well-known symptoms but the description of finger agnosia in 1888 by Jules Badal had escaped the attention of neurologists, a fact which is not altogether surprising in view of the circumstances that the Bordeaux eye specialist published in an ophthalmological journal, and the finger agnosia which he quite clearly described was only one of a large number of deficits shown by his patient. In 1924, Gerstmann once again described finger agnosia, designating it as "a circumscribed disorder of $\frac{\overline{\frac{\sigma}{5}}}{\frac{0}{}}$ orientation to one's own body". In 1927, he $\stackrel{\mathbb{Q}}{\Omega}$ advanced the idea that finger agnosia and agraphia os formed "a new syndrome". However, in 1930 he enlarged the syndrome to include right-left disorientation and acalculia and proposed that it had a highly specific neuropathological significance. At the same time, he discussed the question of the Grundstörung responsible for this presumably "natural" concurrence of behavioural deficits.

Clinical experience appeared to confirm the real i existence of the syndrome; case reports of patients manifesting it appeared in the literature and specula- $\sigma$ tions regarding the basic impairment underlying its occurrence were offered. However, it was certain not rare to encounter patients who showed one, twe or three deficits but not the full syndrome. Whip such observations were often interpreted as simple $\vec{\oplus}$ representing formes frustes of the syndrome, the did indicate at least that the four symptoms did not necessarily occur together in every case and they did have implications regarding the cogency of those theoretical formulations which had been advanced to account for an obligatory concurrence of the deficits. On the other hand, a wide variety of other deficits were also observed to occur in connexion with any or all of the four symptoms. These were given a subordinate status as accompanying symptoms which might or might not be manifested. Among them were general mental impairment, aphasic disorders, colour agnosia, visual disorientation, dyslexia, and constructional apraxia. Indeed, the last-named deficit, constructional apraxia, has been observed so often to accompany one or more of the four deficits that some authors have come to consider it, explicitly or implicitly, as part of the syndrome. For example, Ajuriaguerra and Hécaen (1960), discussing the interrelations of the elements of the syndrome and the connexions with other $N$ symptoms, remark that "the most frequent and most " important association is that between finger agnosia 0 and constructional apraxia. We have seen that such $c$ an association cannot be considered to be simply fortuitous or anatomically conditioned". 
If the "Gerstmann syndrome" is a naturally occurring combination of interrelated deficits, all of which are expressions of a single basic impairment, and not merely the product of selective attention and observation, the four behavioural deficits which comprise it should show stronger "associative bonds", so to speak, among themselves than they do with symptoms which are considered to be outside of the syndrome. If unbiased analysis should show this to be the case, the findings could be reasonably interpreted as evidence for a special coherence of the four symptoms and both the designation of the combination as a syndrome and a search for the common factor responsible for the coherence are justified. On the other hand, if such analysis should disclose that this is not the case, the findings could be reasonably interpreted as indicating that this particular combination of deficits is no more or less likely to occur in patients with cerebral disease than a great many similar combinations and that, unless it can be shown to have a distinctive neuropathological significance, neither its designation as a syndrome nor the postulation of a common psychoneurological factor to account for it (as distinguished from other combinations) is justified.

It might be thought that a comprehensive survey of the pertinent clinical literature would provide the answer to this question of the phenomenological reality of the Gerstmann syndrome. However, the literature proves to be virtually without value in this respect. The methods and criteria utilized for making clinical judgments regarding the presence or absence of defect in the performances investigated are so diverse that the findings from one case to another are not comparable. Not infrequently the criteria employed in making judgments of deficit are not mentioned and notations such as "slight finger agnosia", "questionable right-left disorientation", or "no noteworthy constructional apraxia" are made. Moreover, the majority of case reports fail to mention the presence or absence of one or more pertinent accompanying deficits and hence must be excluded from a systematic analysis. Finally, the factor of selective attention and observation when one is dealing with an already established entity, such as the Gerstmann syndrome, may introduce a bias in the original observations for which the reviewer cannot correct.

It seems evident that the question of the status of the Gerstmann syndrome as a behavioural phenomenon can be answered only by an investigation which has been deliberately designed to answer it and which employs explicitly described procedures for assessment and criteria for judgment. The present paper reports such an investigation.

\section{Case Material and Methods}

The case material consisted of 100 non-psychotic patients with unequivocal diagnoses of disease or injury involving the cerebral hemispheres who had been seen in the neurological and neurosurgical services of the University Hospitals and Veterans Administration Hospital, Iowa City. Any patient who was suffering from acute illness, whose behavour raised the question of psychosis, who had a history of treatment in hospital for a psychiatric disorder, or whose history was suggestive of a state of mental defect dating back to childhood, was excluded from the study. All the patients were between the ages of 16 and 64 years and were clearly capable of understanding directions, cooperating in the test procedures and undergoing one to two hours of examination without discomfort. The mean age of the group was 42 years. The mean educational level was 10 years (range: 5 to 16 years). A variety of cerebral conditions, focal and diffuse, was represented in the group. The performances of 12 right-handed patients with focal disease involving the left parietal lobe were analysed separately, in addition to the main analyses of the total group of 100 patients of which they formed a part.

A control group of 100 non-psychotic patients from the neurological, neurosurgical, and medical services who showed no evidence or history of cerebral disease or injury were also examined, their performances being utilized to establish objective normative standards on the basis of which the presence or absence of impairment could be defined. The mean age of this group was 41 years (range: 16 to 65 years) and mean educational level was 10 years (range: 5 to 14 years).

All the patients were given a battery of seven tests designed to provide objective and comprehensive assessments of the following capacities: (1) right-left orientation; (2) finger localization; (3) arithmetic calculation; (4) writing; (5) constructional praxis; (6) reading; (7) visual memory. The testing procedures were as follows:

Right-left Orientation.-This test battery ( 32 items) required the patient to execute localizing movements to oral command and assessed the following aspects of right-left orientation: (A) with the aid of vision, identification of single lateral body parts on one's own body; (B) with the aid of vision, execution of double uncrossed and crossed commands with respect to lateral body parts on one's own body, e.g., left hand on right eye; (C) without the aid of vision, identification of single lateral body parts; (D) without the aid of vision, execution of double commands; (E) identification of single lateral body parts on a front-view representation of a man; (F) execution of double commands involving identification of lateral body parts of both the patient and the representation, e.g., patient's right hand on a man's right eye. The details of this test battery may be found in a paper by Benton and Kemble (1960). Level of performance was defined as the number of correct localizations made by the patient.

Finger Localization.-This test battery ( 80 items) assessed the following aspects of finger identification: (A) with the aid of vision, presentation of single fingers 
on oral command; (B) identification of single fingers on schematic drawings of the hands; $(C)$ with the aid of vision, identification of single fingers which had been touched; (D) without the aid of vision, identification of single fingers which had been touched; (E) without the aid of vision, identification of pairs of fingers subjected to simultaneous tactile stimulation. (For details regarding the arrangements for tactile stimulation of the fingers, see Benton, 1959.) Level of performance was defined as the number of correct localizations made by the patient.

Arithmetic Calculation.-This untimed 16-item written test consisted of simple problems in addition, subtraction, multiplication, and division involving one- and twodigit numbers. Level of performance was defined as the number of correct solutions.

Writing.-The patient was asked to write his name, copy two simple sentences, write two simple sentences to dictation, and write down anything that came into his mind. Unlined $5 \frac{1}{2} \times 8$ in. paper was used. Each specimen of writing was independently rated by two examiners on the basis of the following factors: alignment of lines; elisions and omissions; formation of letters; spelling; substitutions and additions. Each of these factors was rated from 0 (poor) to 2 (within normal limits). Level of performance was defined in terms of an overall rating consisting of the sum of the two examiners' ratings on each of these factors. However, in the case of patients with sensorimotor deficits of the preferred hand, the "formation of letters" factor was excluded from consideration, and a weighted sum of the examiners' ratings on the other factors was computed.

Constructional Praxis.-Both two-dimensional and three-dimensional praxis was assessed. The twodimensional task consisted in having the patient copy six "stick" designs presented by the examiner while the three-dimensional task consisted in having him copy four models made of rectangular blocks of various sizes and dimensions. The patient was allowed to use only one hand in arranging the sticks or blocks. Level of performance was defined as the number of sticks and blocks correctly placed.

Reading.-The patient read four short paragraphs and, with the paragraphs in view, answered questions which assessed his understanding of them. Level of performance was defined as the number of questions correctly answered. No time limit was enforced.
Visual Memory.-Multiple choice form $G$ of the visual retention test (Benton, 1953) consisting of 15 designs was used. Each design was presented to the patient for 5 secen after which he was required to identify the design on card containing it and three similar designs. Level of performance was defined as the number of correse choices made by the patient.

Distributions of the scores of the 100 control patienţ on each of the seven tests were made and inspected. Fo each distribution, a score which was equalled or exceeded by 91 to $94 \%$ of the patient's was selected as a "cuttin score" and scores below this level were considered to indicate significantly defective performance. Natury "breaks" which occurred in the lower ends of some of the distributions were utilized in establishing these cutting scores, a circumstance which accounts for the sligh variation in the percentage values of the various scores

\section{The Investigation}

Relationships among Performances.-The intef $\overrightarrow{\mathrm{N}}$ correlations among the seven performances for the total group of 100 brain-damaged patients are show in Table I. These are "second-order" correlation coefficients, from which the effects of variation ip age and educational level on performance have been eliminated. Inspection of this matrix suggests that the correlations among the elements of the syndrome are neither higher nor lower than those betwoen these elements and the performances which pres sumably do not belong to the syndrome. Thus, whife the highest correlation coefficient in the mafrid $(0.62)$ is between two syndrome performarees (right-left orientation vs. finger localization), it will be seen that the lowest correlation coefficient $(0.35$ is also between two syndrome performances (finge localization vs. calculation). The mean correlatio coefficient of the performances within the syndrom (derived from averaging the six relevant correlation coefficients) is 0.48 . This may be compared with the mean correlation coefficient between the syndrome performances and those performances which ar outside of the syndrome. This statistic (derived from averaging the 12 relevant correlation coefficients is 0.52 , indicating that the syndrome performances show no closer relationship among themselves thai

TABLE I

SECOND-ORDER CORRELATION COEFFICIENTS (AGE AND EDUCATION "PARTIALLED OUT") IN 100 BRAINDAMAGED PATIENTS

\begin{tabular}{|c|c|c|c|c|c|c|}
\hline & $\begin{array}{c}\text { Finger } \\
\text { Localization }\end{array}$ & Writing & Calculation & $\begin{array}{c}\text { Constructional } \\
\text { Praxis }\end{array}$ & Reading & Visual Memory \\
\hline $\begin{array}{l}\text { Right-left } \\
\text { Finger localization } \\
\text { Writing } \\
\text { Calculation } \\
\text { Constructional praxis } \\
\text { Reading }\end{array}$ & 0.62 & $\begin{array}{l}0.48 \\
0.52\end{array}$ & $\begin{array}{l}0.46 \\
0.35 \\
0.46\end{array}$ & $\begin{array}{l}0.51 \\
0.60 \\
0.44 \\
0.51\end{array}$ & $\begin{array}{l}0.48 \\
0.42 \\
0.57 \\
0.60 \\
0.42\end{array}$ & $\begin{array}{l}0.57 \\
0.50 \\
0.52 \\
0.58 \\
0.47 \\
0.56\end{array}$ \\
\hline
\end{tabular}


TABLE II

MEAN CORRELATION COEFFICIENTS BETWEEN INDIVIDUAL PERFORMANCES AND THE SYNDROME IN 100 BRAIN-DAMAGED PATIENTS

\begin{tabular}{lll}
\hline & $r$ \\
\hline Right-left vs. finger localization - writing - calculation & $0 \cdot 52$ \\
Finger localization vs. right-left - writing - calculation & $0 \cdot 51$ \\
Writing vs. right-left - finger localization - calculation & $0 \cdot 49$ & $0 \cdot 42$ \\
Calculation vs. right-left - finger localization - writing & $0 \cdot 52$ & $0 \cdot 52$ \\
Constructional praxis vs. right-left - finger localization - writing - calculation & $0 \cdot 54$ \\
Reading vs. right-left - finger localization - writing - calculation & \\
Visual memory vs. right-left - finger localization - writing - calculation & \\
\hline
\end{tabular}

they do with performances outside of the syndrome.

A more detailed analysis of the strength of the relationships existing among elements of the syndrome as compared with that existing between them and the other performances is presented in Table II, which shows the mean correlation coefficient of each syndrome performance with the other three syndrome performances and of each extra-syndrome performance with the four syndrome performances. The findings indicate quite clearly that constructional praxis, reading, and visual memory are as closely related to the elements of the syndrome as the latter are among themselves, i.e., that the elements of the syndrome do not show particularly close associative bonds.

Cohesiveness of Syndromes.-As has already been noted, the mean intercorrelation among the elements of the syndrome is 0.48 , which may be interpreted as an index of its "cohesiveness", i.e., of the tendency of elements to vary concurrently. Thirty-five combinations of four performances each can be formed from the seven "parietal" performances investigated in this study. Each combination will include one or more elements of the syndrome, one of them being the syndrome. If there is a special tendency for the elements of the syndrome to vary concurrently, its observed mean intercorrelation of 0.48 should be higher than those derived from combinations consisting of mixtures of syndrome and nonsyndrome elements. The mean correlation coefficient (derived from the six relevant correlation coefficients) of each of the 34 mixed combinations was computed. They ranged in size from 0.48 to 0.54 . Two coefficients were as high as 0.54 and four were as low as $\mathbf{0 . 4 8}$. It is clear from these findings that all 35 combinations of elements show about the same degree of cohesiveness and that the syndrome is not at all distinctive in this respect.

The incidence of significant deficit in performance (as defined above, i.e., performances which fell within the lowest 6 to $9 \%$ of the scores of the control group) was computed for each patient. Twenty-nine showed no significant deficits and 23 showed only one deficit. The remaining 48 patients showed two to seven deficits, 10 of them showing four deficits. Examination of these 10 sets of four deficits disclosed that all involved combinations of syndrome and non-syndrome performances. The pure Gerstmann syndrome did not appear. The combination of deficits in finger localization, writing, reading, and constructional praxis appeared twice. Eight other combinations appeared once. Again the findings indicate no particularly strong tendency for the symptoms of the Gerstmann syndrome to appear concurrently.

Performances of Patients with Left Parietal Lesions.-It might be argued that assumptions about the essential unity of the elements of the Gerstmann syndrome hold only for patients with parieto-occipital disease of the dominant hemisphere and that a study involving patients with various types of lesion cannot provide cogent evidence about these assumptions. This contention is quite debatable. The explanations which have been advanced to account for the supposedly obligatory concurrence of the symptoms have been of a broad psychological or psychoneurological nature and have no necessary reference to a specific locus of the responsible lesion. Thus such concepts as the importance of intact finger gnosis for writing and calculation, of intact rightleft orientation for calculation, or of intact spatial thinking for all the performances have been applied to all situations in which the deficits appear, e.g., developmental deficit, and are not dependent upon the presence of a specified lesion. Nevertheless, it seemed worthwhile to make a separate analysis of the performances of the 12 right-handed patients in the group who had disease of the left parietal lobe to determine the nature of the relationships of the syndrome performances among themselves and with the other performances.

The mean correlation coefficient of each of the syndrome performances with the other three syndrome performances and of each extra-syndrome performance with the four syndrome performances is shown in Table III. It is evident from inspection of this table that constructional praxis, reading, and visual memory are as closely related to the elements 
TABLE III

MEAN CORRELATIONS BETWEEN INDIVIDUAL PERFORMANCES AND THE SYNDROME IN 12 PATIENTS WITH LEFT PARIETAL LOBE DISEASE

Right-left vs. finger localization - writing - calculation

Finger localization vs. right-left - writing - calculation

Writing vs. right-left - finger localization - calculation

Writing vs. right-left - finger localization - calculation

Constructional praxis vs. right-left - finger localization - writing - calculation

Constructional praxis vs. right-left - finger localization - writing

Visual memory vs. right-left - finger localization - writing - calculation

of the syndrome as the latter are to each other. Thus in this group of patients with focal disease of the left parietal lobe there is also no evidence that the elements of the syndrome show any noteworthy strength of association.

The significant deficits in performance shown by these 12 patients are shown in detail in Table IV. It will be seen that no patient showed the full Gerstmann syndrome. Four patients (E.H., R.K., A.P., D.T.) presented with three Gerstmann symptoms but in each case these appeared in association with other deficits.

\section{Discussion}

This investigation represents an attempt to determine whether the four behavioural deficits comprising the so-called "Gerstmann syndrome" form a distinctive constellation. A variety of analyses, which provided evidence concerning the strength of the relationships existing among these elements and that existing between the elements and performances which do not belong to the syndrome, were done. These analyses, made both on a large group of patients with diverse types of cerebral disease and a smaller group of right-handed patients with focal disease of the left parietal lobe, consistently indicated that the particular combination of behavioural deficits which form the syndrome show no stronger internal associative bonds than do a score of other combinations of behavioural deficits. A large number of combinations of four behavioural defici may be presented by patients with cerebral disease The analyses of the present study suggest that the is about an equal probability of occurrence for ang one of them, including the combination known, as the Gerstmann syndrome.

These results provide a reasonable explanation $\overrightarrow{\text { of }}$ the frequent reports in the clinical literature of s@ called incomplete forms of the syndrome as well $\overline{3}$ the even more frequent reports of symptoms which are observed to "accompany" it. Such behaviour pictures are simply other combinations of deficitis which tend not to be accepted in their own right because of the predilection of clinical observers to look first for elements of the Gerstmann combination and to use these elements as an anchoring poip to which accompanying symptoms may be attacheg The findings also indicate that theoretical specinlations concerning the basic impairment which nas underlie the so-called Gerstmann syndrome Fare hardly warranted. There is no obligatory cong currence of deficits to account for and, in any case if it is necessary to explain this particular combinatio of deficits, it is equally necessary to explain the existence of a score of other combinations behavioural deficits.

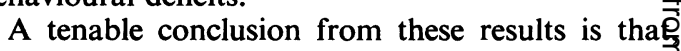
judged from the standpoint of behavioural analysis, the Gerstmann syndrome is a fiction; it is simply a artifact of defective and biased observations. Objes

TABLE IV

INCIDENCE OF DEFICITS IN PATIENTS WITH LEFT PARIETAL LOBE DISEASE

\begin{tabular}{|c|c|c|c|c|c|c|c|c|}
\hline No. & Patient & Right-left & $\begin{array}{c}\text { Finger } \\
\text { Localization }\end{array}$ & Writing & Calculation & $\begin{array}{c}\text { Constructional } \\
\text { Praxis }\end{array}$ & Reading & $\begin{array}{c}\text { Visual } \\
\text { Memory }\end{array}$ \\
\hline $\begin{array}{r}1 \\
2 \\
3 \\
4 \\
5 \\
6 \\
7 \\
8 \\
9 \\
10 \\
11 \\
12\end{array}$ & $\begin{array}{l}\text { N.F. } \\
\text { E.H. } \\
\text { V.H. } \\
\text { R.K. } \\
\text { E.K. } \\
\text { A.P. } \\
\text { D.T. } \\
\text { E.H. } \\
\text { R.M. } \\
\text { E.M. } \\
\text { J.C. } \\
\text { J.D. }\end{array}$ & $\begin{array}{l}0 \\
+ \\
0 \\
+ \\
0 \\
+ \\
+ \\
0 \\
0 \\
0 \\
+ \\
0\end{array}$ & $\begin{array}{l}\text { o } \\
+ \\
\text { o } \\
+ \\
\text { o } \\
+ \\
\text { o } \\
0 \\
0 \\
0 \\
+ \\
\mathbf{0}\end{array}$ & $\begin{array}{l}\text { o } \\
0 \\
0 \\
0 \\
0 \\
0 \\
+ \\
0 \\
0 \\
0 \\
0 \\
0\end{array}$ & $\begin{array}{l}0 \\
+ \\
0 \\
+ \\
0 \\
+ \\
+ \\
0 \\
0 \\
0 \\
0 \\
0\end{array}$ & $\begin{array}{l}+ \\
+ \\
0 \\
+ \\
+ \\
+ \\
0 \\
0 \\
0 \\
0 \\
+ \\
0\end{array}$ & $\begin{array}{l}\text { o } \\
\text { o } \\
\text { o } \\
+ \\
\mathbf{o} \\
+ \\
+ \\
\mathbf{o} \\
\mathbf{0} \\
\mathbf{0} \\
\mathbf{0} \\
\mathbf{o}\end{array}$ & $\begin{array}{l}+ \\
+ \\
0 \\
+ \\
\mathbf{0} \\
\mathbf{0} \\
\mathbf{0} \\
\mathbf{0} \\
\mathbf{0} \\
\mathbf{0} \\
\mathbf{0} \\
\mathbf{0}\end{array}$ \\
\hline
\end{tabular}

$(+=$ Deficit; $0=$ no deficit $)$ 
tive, unbiased observation discloses a large number of combinations of parietal deficits. Since all these combinations appear to be about equally strong a vith respect to their internal associative bonds and frequency of occurrence, either all or none should be designated as syndromes.

This study has not been concerned directly with the question of the possible neuropathological significance of the combination of deficits known as the Gerstmann syndrome. Despite the fact that this - combination of deficits does not seem to be any different from many other combinations of parietal symptoms, there is the possibility that when it is manifested in pure form it does possess the highly specific focal diagnostic significance which has been claimed for it, namely, a lesion of "that nodal area which corresponds to the angular gyrus in its transition to the second occipital convolution" (Gerstmann, 1957). However, Critchley (1953), reviewing the clinical literature, was inclined to conclude that the syndrome was more reasonably interpreted as a sign of parietal disease in general rather than specifically indicating a lesion of the angular gyrus. Similarly, Heimberger, DeMyer, and Reitan (1957) found that when patients manifested the full syndrome, the responsible lesion, even when restricted to the dominant hemisphere, was invariably an extensive one. Finally, there are case reports, such as the recent one by Brusa, Rossi, and Tartarini (1960), of the occurrence of the syndrome in patients with lesions apparently restricted to the frontal lobes. Thus those analyses of the question as have been done throw considerable doubt on the assertion that the syndrome has a highly specific focal diagnostic import. If it does possess such a significance, it might be expected that the other 34 of the 35 syndromes investigated in this study would also have a similarly specific significance. But the weight of clinicopathological evidence gathered over the last 60 years makes it extremely unlikely that such "punctate" localization of functions or deficits exists.

In directing the attention of neurologists both to certain disorders of the body schema and to the unusual variety of combinations of behavioural deficits which a brain-damaged patient may show, the Gerstmann syndrome has perhaps been a useful fiction during the past 30 years. Now that these purposes have been accomplished, adherence to the fiction may well operate to retard further advances in the understanding of the patterns of behavioural deficit which may occur as a consequence of cerebral disease, for it tends to prejudice clinical observation and to produce a distorted picture of the organization of abilities and disabilities in brain-damaged patients.

\section{Summary}

Systematic, objective analysis of the performances of patients with cerebral disease on seven "parietal" tasks (right-left orientation, finger localization, writing, calculation, constructional praxis, reading, visual memory) indicates that many combinations of deficits, including that known as the "Gerstmann syndrome", may be observed. The syndrome appears to be no different from the other combinations in respect to either the strength of the mutual interrelationships among its elements or the strength of the relationships between its elements and performances not belonging to it. These results hold both for patients with diverse cerebral conditions and for those with focal lesions of the dominant parietal lobe.

The findings are interpreted as indicating that the Gerstmann syndrome is an artifact of defective and biased observation. Further, a review of the pertinent clinical literature offers little support for its alleged focal diagnostic significance. The general conclusion is that the syndrome is a fiction which has perhaps served a useful purpose in the past in certain respects, but which now carries the hazard of retarding advances in the understanding of the organization of abilities and disabilities in patients with cerebral disease.

This investigation was supported by a grant (B-616) from the National Institute of Neurological Diseases and Blindness, U.S. Public Health Service.

\section{REFERENCES}

Ajuriaguerra, J. de, and Hécaen, H. (1960). Le Cortex Cérébral. Masson, Paris.

Badal, J. (1888). Arch. Ophtal. (Paris), 8, 97.

Benton. A. L. (1953). Manuel pour l'Application clinique du Test de Rétention Visuelle. Editions du Centre de Psychologie Appliquée, Paris.

(1959). Right-Left Discrimination and Finger Localization: Development and Pathology. Hoeber, New York.

and Kemble, J. D. (1960). Psychiat. et Neurol. (Basel), 139, 49.

Brusa, A., Rossi, R., and Tartarini, E. (1960). Encéphale, 49, 319.

Critchley, M. (1953). The Parietal Lobes. Arnold, London.

Gerstmann, J. (1924). Wien klin. Wschr., 37, 1010.

(1927). Z. ges. Neurol. Psychiat., 108, 152. (1930). Nervenarzt., 3, 691.

(1930). Nervenarzt., 3, 691.

Heimberger, R. F., DeMyer, W., and Reitan, R. M. (1957). Paper read at the American Academy of Neurology, Boston. 